\title{
Erratum to: Biosurfactant production by AL 1.1, a Bacillus licheniformis strain isolated from Antarctica: production, chemical characterization and properties
}

\section{Jonathan Coronel-León ${ }^{1}$ • Guillermo de Grau ${ }^{1}$ - Ariadna Grau-Campistany ${ }^{2}$.}

Maribel Farfan $^{1}$ - Francesc Rabanal ${ }^{2}$ - Angels Manresa ${ }^{1}$ • Ana María Marqués ${ }^{1}$

Published online: 10 April 2015

(C) Springer-Verlag Berlin Heidelberg and the University of Milan 2015

Erratum to: Ann Microbiol

DOI 10.1007/s13213-015-1045-x

The original version of this paper unfortunately contained error. Figure $1 \mathrm{~B}$ is incorrect and should be replaced by the following Figure 1B:

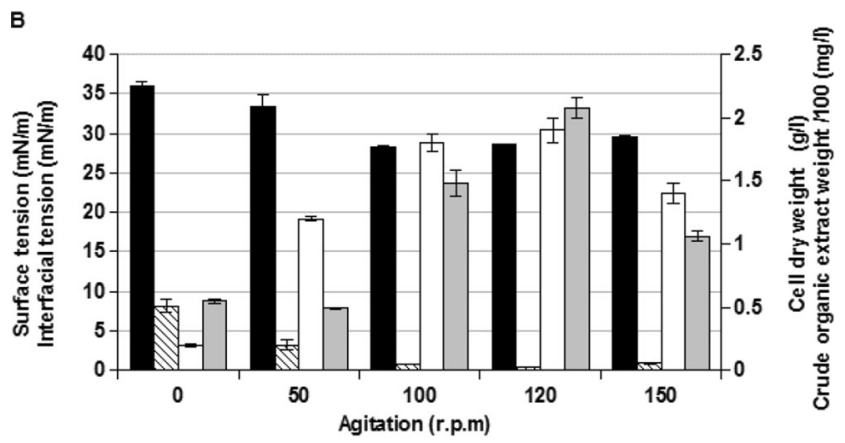

- Supernatant Surface tension $\mathbb{Q}$ Supernatant Interfacial tension

$\square$ Cells dry weight $\quad \square$ Crude organic extract weight

The online version of the original article can be found at http://dx.doi.org/ 10.1007/s13213-015-1045-x.

Ana María Marqués ammarques@ub.edu

Laboratori de Microbiologia, Facultat de Farmàcia, Universitat de Barcelona, 08028 Barcelona, Spain

2 Departament de Química Orgànica, Facultat de Químiques,

Universitat de Barcelona, 08028 Barcelona, Spain 